\title{
Embolic Stroke of undetermined Source in juvenile stroke patients: multinational data from three tertiary care centers
}

Alexandra Prakapenia, Eskandari Ashraf, George Ntaios, Konstantinos Vemmos, Davide Strambo, Lars-Peder Pallesen, Jessica Barlinn, Timo Siepmann, Patrik Michel, Volker Puetz, Kristian Barlinn

\section{Dresden University Stroke Center, Department of Neurology, Carl Gustav Carus University Hospital, University of} Technology Dresden, Dresden, Germany

\section{BACKGROUND}

$>$ Embolic stroke of undetermined source (ESUS) may be less common in juvenile than in elderly patients.

\section{AIMS}

To explore the frequency of ESUS in patients with juvenile stroke and its predictive role on stroke outcomes

\section{METHODS}

$>$ Patients with acute cerebral ischemia aged 18-45 years admitted between 1992 and 2015 were studied

Data from three tertiary care hospitals (Lausanne, Athens, Dresden) were analyzed

$>$ Stroke etiology was determined using TOAST and ESUS criteria [4]

$>$ Predictors of stroke outcomes (multivariable logistic regression model): $(\mathrm{mRS} \leq 2)$

$\rightarrow$ functional outcome at discharge $\rightarrow$ in-hospital death

\section{RESULTS}

702 patients with juvenile acute ischemic stroke (Figure 1)

Etiology:

- 35,5\% non-identifiable cause

$$
\rightarrow 17,4 \% \text { ESUS }
$$

- $64,5 \%$ other identifiable cause

$\rightarrow 30,6 \%$ miscellaneous

$\rightarrow 22,1 \%$ cardio-embolic

$\rightarrow 6,1 \%$ large-vessel

$\rightarrow 5,7 \%$ small-vessel

ESUS vs. none-ESUS: except for arterial hypertension (12,3\% vs. $20,9 \%$ ), no differences regarding sex, age, vascular risk factors, baseline stroke severity

ESUS: independent predictor of favorable functional outcome at discharge (OR: 2.94; $95 \% \mathrm{Cl}, 1.36-6.38, \mathrm{p}=0.006$ )

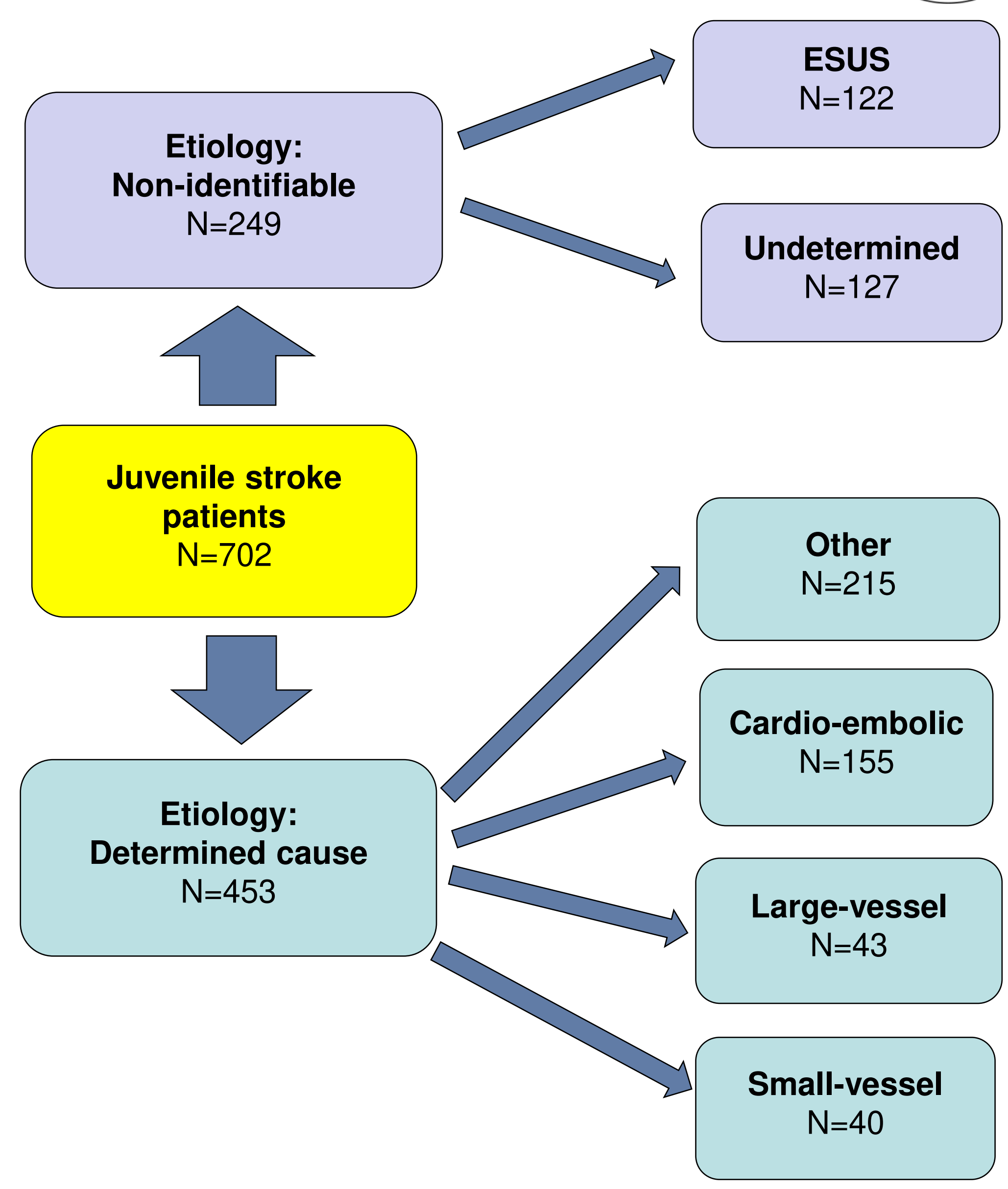

Figure 1. Study flow chart

\section{CONCLUSIONS}

$\checkmark E S U S$ is relatively frequent in a multinational cohort of juvenile (18-45y) stroke patients

$>$ Predictive role of ESUS on functional outcome in young stroke patients is not well studied und may differ from those with identifiable stroke causes

\section{LITERATURE}

[1]. Ntaios G, Papavasileiou V, Milionis H, Makaritsis K, Manios E, Spengos K, et al. Embolic strokes of undetermined source in the athens stroke registry: A descriptive analysis. Stroke. 2015:46:176-181; [2]. Ntaios G, Papavasileiou V, Milionis H, Makaritsis K, Vemmou A, Koroboki E, et al. Embolic strokes of undetermined source in the athens stroke registry: An outcome analysis. Stroke. 2015;46:2087-2093; [3]. Michel P, Odier C, Rutgers M, Reichhart M, Maeder P, Meuli R, et al. The acute stroke registry and analysis of lausanne (astral): Design and baseline analysis of an ischemic stroke registry including acute multimodal imaging. Stroke. 2010;41:2491-2498; [4] Michel P, Odier C, Rutgers M, Reichhart M, Maeder P, Meuli R, et al. The acute stroke registry and analysis of lausanne (astral): Design and baseline analysis of an ischemic stroke registry including acute multimodal imaging. Stroke. 2010;41:2491-2498 